\title{
Changes of quantitative CT-based airway wall dimensions in patients with COVID-19 during early recovery
}

\author{
Jiaxuan $\mathrm{Xu}^{1 \#}$, Zhenyu Liang ${ }^{1 \#}$, Wenhua Jian ${ }^{1 \#}, \mathrm{Jianyu}_{\mathrm{Li}^{2}}$, Guoyan Tang ${ }^{1}$, Xiaoneng Mo ${ }^{3}$, \\ Dongying Zhang', Jinping Zheng ${ }^{1}$, Yuanxin Qian ${ }^{2}$, Jinxin Liu ${ }^{4}$, Shiyue $\mathrm{Li}^{1}$
}

\begin{abstract}
${ }^{1}$ China State Key Laboratory of Respiratory Disease, National Clinical Research Center for Respiratory Disease, Guangzhou Institute of Respiratory Health, First Affiliated Hospital of Guangzhou Medical University, Guangzhou, China; ${ }^{2}$ Department of Radiology, the First Affiliated Hospital of Guangzhou Medical University, Guangzhou, China; ${ }^{3}$ Department of Respiratory Medicine, Guangzhou Eighth People's Hospital, Guangzhou, China; ${ }^{4}$ Department of Radiology, Guangzhou Eighth People's Hospital, Guangzhou Medical University, Guangzhou, China

Contributions: (I) Conception and design: J Xu, Z Liang; (II) Administrative support: D Zhang, J Zheng, S Li; (III) Provision of study materials or patients: J Liu, X Mo; (IV) Collection and assembly of data: W Jian, G Tang; (V) Data analysis and interpretation: J Li, Y Qian; (VI) Manuscript writing: All authors; (VII) Final approval of manuscript: All authors.

"These authors contributed equally to this work.

Correspondence to: Shiyue Li. State Key Laboratory of Respiratory Disease, National Clinical Research Center for Respiratory Disease, Guangzhou Institute of Respiratory Health, the First Affiliated Hospital of Guangzhou Medical University, Guangzhou 510120, China. Email: lishiyue@188.com; Jinxin Liu. Department of Radiology, Guangzhou Eighth People’s Hospital, Guangzhou Medical University, Guangzhou 510060, China. Email: liujx83710378@126.com; Yuanxin Qian. Department of Radiology, the First Affiliated Hospital of Guangzhou Medical University, Guangzhou 510120, China. Email: qian.yuanxin@163.com.
\end{abstract}

Background: As the coronavirus disease 19 (COVID-19) pandemic evolves, the need for recognizing the structural pulmonary changes of the disease during early convalescence has emerged. Most studies focus on parenchymal destruction of the disease; but little is known about whether the disease affects the airway. This study was conducted to investigate the changes in airway dimensions and explore the associated factors during early convalescence in patients with COVID-19.

Methods: We retrospectively analyzed quantitative computed tomography (CT)-based airway measures of 69 patients with COVID-19 from 5 February to 17 March 2020, and 32 non-COVID-19 participants from 1 January 2018 to 31 December 2019 from Guangzhou, China. The well-established measures of wall area fraction and the square root of the wall area of a hypothetical bronchus with an inner perimeter of $10 \mathrm{~mm}$, were used to describe airway wall dimensions. We described the characteristics of the dimensions and inner area of airways in 66 patients with COVID-19 at the initial and convalescent stages of the disease, and compared them with the non-COVID-19 group. Linear regression models were constructed to investigate the association of airway dimensions with duration of hospitalization or disease severity after recovery. Partial correlation coefficients were calculated to investigate whether inflammatory markers were related to airway dimensions.

Results: Among 66 patients with COVID-19, airway dimensions were greater during disease initiation than early convalescence, which was significantly greater than in non-COVID-19 participants. No significant difference was found between the patients with COVID-19 at the initial stage and the non-COVID-19 controls regarding the first to eighth generations of the inner area. In adjusted regression models, duration of hospitalization was negatively associated with wall area fraction of the first to the sixth generation of airways. No significant associations exist between airway dimensions and disease severity, or airway dimensions with inflammatory markers.

Conclusions: Airway dimensions in patients with COVID-19 during disease initiation are greater than those in non-COVID-19 participants. Such structural airway changes continue to remain significantly greater during early convalescence. No evidence shows that disease severity or inflammatory markers are 
associated with airway dimensions, implying that the primary lesion attacked by COVID-19 might not be the airways.

\begin{abstract}
Keywords: Airway wall thickness; coronavirus disease 19 (COVID-19); coronavirus; early convalescence; quantitative computed tomography (quantitative CT)

Submitted Sep 01, 2020. Accepted for publication Jan 20, 2021.

doi: $10.21037 /$ jtd-20-2790

View this article at: http://dx.doi.org/10.21037/jtd-20-2790
\end{abstract}

\section{Introduction}

A novel disease was first recognized in December 2019 (1), and its pathogen was later identified (2) and termed severe acute respiratory syndrome coronavirus 2 (SARS-CoV-2) (3) based on a phylogeny similar to SARS-CoV (4). Since the first case was identified, coronavirus disease 19 (COVID-19) has raged across 216 countries and regions, causing over 13 million confirmed cases and over 500,000 deaths by 15 July 2020 worldwide (5). As the pandemic evolves, awareness of recognizing sequential influences the disease has on the human body after recovery has been aroused.

The human lung is the most affected organ after infection with SARS-CoV-2 (6,7). Thoracic computed tomography (CT) has played a key role in the diagnosis and further evaluation of COVID-19 pneumonia (8-13). The most typical reported abnormalities presented on CT are lung parenchymal destruction, such as ground-glass opacity (GGO), bilateral patchy shadowing and consolidation (14-16). These abnormal CT patterns may persist even after patients with COVID-19 recover and are discharged from the hospital (16). COVID-19 shares many similarities with SARS and MERS in pathogenesis and clinical characteristics (1,4,17-20). Lung fibrosis is known to be a persistent impairment in patients recovered from SARS and MERS and could last for months or even longer (21-24). Therefore, discussion on pulmonary structural changes resulting from SARS-CoV-2 infection is mostly focused on GGO and pulmonary fibrosis. However, whether SARS-CoV-2 causes airway dysfunction, such as airway wall thickening, which may result in irreversible airflow obstruction, has not yet been reported.

Airway wall thickening is usually the result of persistent inflammatory reaction in the bronchi. Previous research has established the quantitative CT-based measures of percentage of wall area (WA\%) and Pi10 to evaluate airway wall thickness. The WA\% is defined as the percentage of airway wall cross-sectional area to total airway cross- sectional area (25). The Pi10 is defined as the square root of the wall area of a hypothetical bronchus with an inner perimeter of $10 \mathrm{~mm}$ (26). In patients with chronic obstructive pulmonary disease, airway dimension is found to be associated with impaired respiratory-related quality of life and airflow limitation $(27,28)$.

The aim of this analysis is to investigate how airway dimensions change in patients with COVID-19 and explore the associated potential influencing factors using quantitative CT-based measurement. We hypothesize that airway dimensions in patients with COVID-19 during disease initiation, including the WA\% of the $1^{\text {st }}$ to $8^{\text {th }}$ generations of airway as well as Pi10, are greater than nonCOVID-19 participants, and that such airway structural changes cannot be fully recovered in early convalescence. We present the following article in accordance with the STROBE guidelines checklist (available at http://dx.doi. org/10.21037/jtd-20-2790).

\section{Methods}

\section{Participant selection}

This study was conducted in accordance with the Declaration of Helsinki (as revised in 2013). The study was approved by the Institutional Review Board at both Guangzhou Eighth People's Hospital and the First Affiliated Hospital of Guangzhou Medica University (Ethics No. 20201134). Written informed consent for data collection was waived for all participants. All the data were de-identified to protect the privacy of participants.

In this retrospective observational study, we reviewed the medical records of patients diagnosed with COVID-19 in Guangzhou Eight People's Hospital from 5 February to 17 March in 2020. All the laboratory-confirmed patients with COVID-19 were admitted to the hospital for quarantine and treatment. Diagnosis and clinical severity classification of COVID-19 and discharge decisions were made by 
clinicians as per the guideline released by the National Health Commission of China (8). Participants with typical symptoms or epidemiologic history were considered suspected cases. Suspected cases with serological evidence of SARS-CoV-2 infection were diagnosed as COVID-19 cases. Based on the severity of dyspnea and CT imaging, COVID-19 patients were classified as mild, moderate, severe or critical cases. During hospitalization, effective oxygen therapy, anti-viral therapeutics and corticosteroids were given to patients depending on the severity of their condition. Prognostic indicators, such as C-reactive protein (CRP), blood cell count and arterial blood gas analysis were surveilled. Patients were discharged when dyspnea and chest imaging were greatly improved, body temperature returned to and remained normal for at least 3 days and two consecutive viral nucleic acid nasopharyngeal swabs or sputum samples were negative. Among the COVID-19 cases reviewed, those who had recovered from the disease and had gone through high-resolution CT scans during convalescence were included in this study. Patients who did not fulfill CT scanning protocol compliance requirements for the quantitative analysis were excluded.

To establish a non-COVID-19 participant group for comparison, we searched the electronic medical record (EMR) of outpatients who had visited the First Affiliated Hospital of Guangzhou Medica University from 1 January 2018 to 31 December 2019. For the current study, nonCOVID-19 participants were randomly selected based on the inclusion criteria: (I) above 18 years old; (II) with normal high-resolution CT scans based on visual assessment to rule out major pulmonary diseases; (III) without airflow obstruction or small airway dysfunction based on pulmonary functional testing (PFT) performed at the same time as the high-resolution CT scanning; and (IV) without a history of wheezing or asthma, or severe systemic abnormalities. Participants who did not fulfill CT scanning protocol compliance requirements for the quantitative analysis were excluded.

\section{Data collection}

Demographic and clinical data were extracted from the $\mathrm{EMR}$, including age, sex, body mass index (BMI), smoking status, and baseline pulmonary abnormality. Additionally, disease severity and duration of hospitalization were gathered for patients with COVID-19, while PFT and CT report records were gathered for non-COVID-19 participants. Original DICOM data were collected for further quantitative assessment.

\section{CT protocol}

Patients at both the initial and convalescent stage of COVID-19 and non-COVID-19 participants had all undergone non-contract thoracic $\mathrm{CT}$ scan at end-inspiration in the supine position, using a 64-slice multi-detector CT scanner (Optima CT680, GE Medical Systems, Milwaukee, WI, USA, for patients with COVID-19; and SOMATOM Definition AS+, Siemens Healthineers, Germany, for nonCOVID-19 participants).

The following acquisition and reconstruction parameters were used: for patients with COVID-19, tube voltage $120 \mathrm{kV}$, slice thickness 0.625 to $1.25 \mathrm{~mm}$ and slice interval 0.625 to $1.25 \mathrm{~mm}$; for non-COVID-19 participants, tube voltage $120 \mathrm{kV}$, slice thickness $1 \mathrm{~mm}$ and slice interval $1 \mathrm{~mm}$.

\section{CT Visual Assessment and Quantitative Analysis}

Quantitative CT analysis was performed using an automated analysis platform, the VIDA version 2.2 (VIDA Diagnostics, Inc., Coralville, IA, USA), to measure the inner area and dimensions of the airway, which included: (I) wall area fraction (WA\%) of the first to eighth generations of airways; (II) Pi10. WA\% was calculated automatically using the formula [(outer area of the airway-lumen area of airway)/outer area of airway] $\times 100 \%(25)$. The Pi10 was automatically predicted using a scatter plot and a regression model (26). The inner area and dimensions of the airway were measured throughout the entire lung of each participant.

\section{Statistical analysis}

Continuous variables were described as mean (standard deviation, $\mathrm{SD}$ ), while categorical variables were described as count (proportion). The chi-square test was used for categorical variables comparison. An independent-sample $t$-test was applied to compare WA\% and Pi10 in patients with COVID-19 versus non-COVID-19 participants and for subgroup comparison. Paired-sample $t$-test was used to compare WA\% and Pi10 in patients with COVID-19 between the initial and convalescent stages. Linear regression analysis was applied to determine the relationship between clinical characteristics, which included duration of hospitalization and disease severity, and quantitative CTbased airway dimensions in patients with COVID-19 at the 
convalescent stage. Partial correlation analysis was used to further investigate the correlation of airway dimensions and inflammation level. All of the multivariate linear regression models and the partial correlation analysis were adjusted for sex, age and smoking status despite statistical significance of univariate analysis. No missing data were imputed. Statistical significance was defined as a $\mathrm{P}$ value of less than 0.05. All of the statistical analyses were performed using SPSS (version 23; SPSS Inc., Chicago, IL, USA).

\section{Results}

\section{Characteristics of participants}

For this analysis, 69 patients with COVID-19 as well as 32 non-COVID-19 control participants were studied. The 32 control participants visited the outpatient department of the hospital and underwent the CT imaging and PFT because they had respiratory symptoms such as cough or phlegm. After they had completed CT imaging, PFT and other relevant medical examinations, those with a diagnosis of pulmonary diseases that could cause bronchial wall thickening, including asthma, chronic obstructive pulmonary disease and bronchiectasis, were excluded. Among 69 patients who went through high-resolution CT scans at both the initial and convalescent stages, one had unqualified CT scans for VIDA analysis and two patients had incomplete CT scans at the initial stage, leaving 66 cases with paired CT scans for comparison of airway dimensions with non-COVID-19 participants. The 69 patients with COVID-19 were on average $49.6 \pm 13.8$ years old, with 36 males (52.2\%), eight smokers (11.6\%) and four with baseline pulmonary abnormalities (5.8\%) (Table 1). The relatively low prevalence of smokers may have been due to selection bias, and in the control group, important reason might have been that many smokers who underwent both chest $\mathrm{CT}$ and pulmonary function test (PFT) were excluded from the study because of the abnormalities found in these two examinations. The five control participants with baseline pulmonary abnormalities were suspected cases of cough-variant asthma, findings of localized fibrotic scarring on chest CT imaging, suspected chronic obstructive pulmonary disease, chronic bronchitis, and suspected chronic obstructive pulmonary disease, respectively. Based on their normal pulmonary function testing and negative bronchial provocation tests, these five participants were included and labeled as control participants with pulmonary abnormalities. The majority of the COVID-19 cases were diagnosed as moderate and severe $(62.3 \%$ and $34.8 \%$, respectively), and no critical patients were included in this study. The average duration of hospitalization was $23.5 \pm$ 8.4 days. The median interval between admission and the first CT scan was 1 day (IQR 1-2) after admission, and the median interval between the second CT scan and discharge was 1.5 days (IQR 1-3). Four patients with COVID-19 demonstrated CT scans with no visually obvious abnormal patterns at the initial stage. One case was missing for the analysis of duration of hospitalization. Basic demographic and clinical data for the 66 patients with paired CT scans and the 32 non-COVID-19 participants are presented in Table 2. The non-COVID-19 participant group was on average younger and roughly matched to the COVID-19 patients with regards to sex, BMI, smoking status, and baseline pulmonary diseases. Of note, the proportion of smokers in both populations was low $(9.4 \%$ versus $12.1 \%$; $\mathrm{P}>0.05$ ), and so was the proportion of baseline pulmonary diseases $(15.6 \%$ versus $4.5 \% ; \mathrm{P}>0.05)$.

\section{Characteristics of quantitative CT-evaluated airway dimensions}

The WA $\%$ of the $1^{\text {st }}$ to $8^{\text {th }}$ generations of airway and the Pi10 were compared between patients with COVID-19 and non-COVID-19 participants (Table 3). Both groups had one case missing for the evaluation of the WA\% of the $8^{\text {th }}$ generation of airway. During disease initiation, the WA\% of the $1^{\text {st }}$ to $8^{\text {th }}$ generations of airways and the Pi10 in patients with COVID-19 were generally greater than those in non-COVID-19 cases. After active supportive treatment during hospitalization, airway dimension measurements significantly decreased as the patients with COVID-19 were in the convalescent stage. Changes in WA\% and Pi10 in individual patients with COVID-19 with paired CT scans at two time points are shown in Figure 1. When comparing airway dimensions of patients with COVID-19 in early convalescence to non-COVID-19 cases, the WA\% of the $1^{\text {st }}$ to $8^{\text {th }}$ generations of airways in the patient group remained significantly greater than that in the non-COVID-19 group, except for the $6^{\text {th }}$ generation of airway $(67.53 \%$ versus $66.79 \%, \mathrm{P}=0.156)$. As for Pi10, however, there was no significant difference between the two groups (3.90 versus $3.91 \mathrm{~mm}, \mathrm{P}=0.631$ ). This result indicated that the thickening of the airway wall in patients with COVID-19 did not fully recover to the normal level for a short period after recovery.

Inner area $\left(\mathrm{mm}^{2}\right)$ of the $1^{\text {st }}$ to $8^{\text {th }}$ generations of airways 
Table 1 Clinical characteristics and quantitative airway dimension of COVID-19 patient subgroups at the convalescent stage

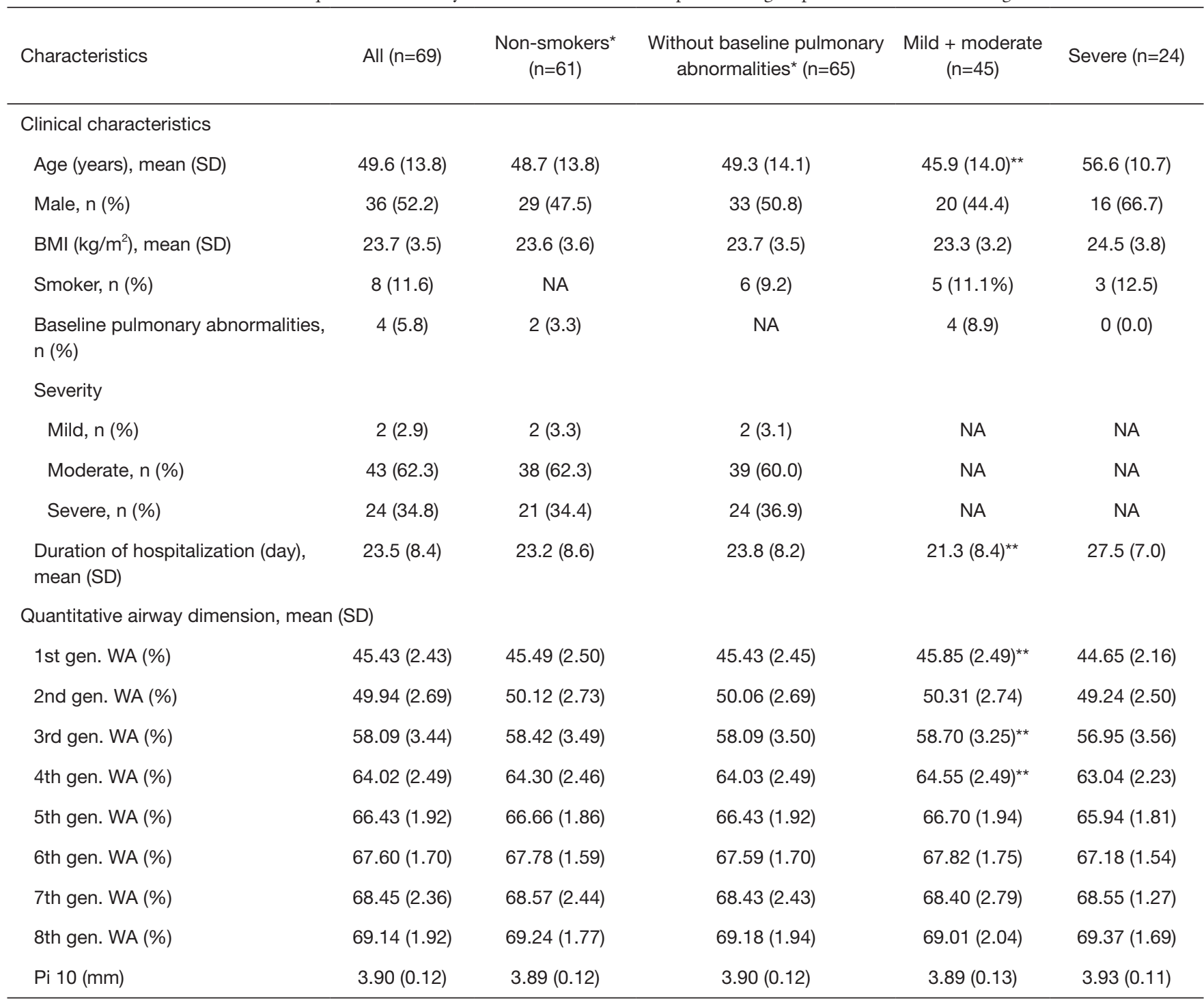

NA, not applicable; WA\%, wall area fraction; gen., generation; Pi10, internal airway perimeter of 10 mm. *, denotes no statistically significant difference $(\mathrm{P}>0.05)$ exists compared to all 69 subjects for all parameters; ${ }^{* *}$, denotes $\mathrm{P}<0.05$ compared to severe subgroup.

between non-COVID-19 controls and patients with COVID-19 were also investigated, to better understand the bronchial structural changes after SARS-CoV-2 infection. Compared to that in non-COVID-19 participants, the inner area of the $1^{\text {st }}$ to $8^{\text {th }}$ generations of airways in patients with COVID-19 patients did not significantly decrease (Table 4).

\section{Characteristics of patient subgroups}

Considering that several factors could affect airway dimensions, such as smoking status, baseline pulmonary diseases and disease severity, the overall COVID-19 patient population was divided into different subgroups, with the purpose of investigating whether WA\% and Pi10 differ in patient subgroups. Results are presented in Table 1. In the non-smoker subgroup, similar demographic and clinical characteristics are presented as the overall population. Neither the WA\% of the $1^{\text {st }}$ to $8^{\text {th }}$ generations of airways nor the Pi10 showed a significant difference between this subgroup and the total population. Among all 69 patients included in the analysis, 65 were without baseline pulmonary abnormalities. Similar characteristics were 
Table 2 Clinical characteristics of patients with COVID-19 who had paired CT scans versus non-COVID-19 subjects

\begin{tabular}{|c|c|c|c|}
\hline Characteristics & COVID-19 (n=66) & Non-COVID-19 $(n=32)$ & $P$ value \\
\hline Male, n (\%) & $33(50.0)$ & $14(43.8)$ & 0.561 \\
\hline BMI $\left(\mathrm{kg} / \mathrm{m}^{2}\right)$, mean (SD) & $23.6(3.5)$ & $24.6(3.6)$ & 0.199 \\
\hline Smoker, n (\%) & $8(12.1)$ & $3(9.4)$ & 1.000 \\
\hline \multicolumn{4}{|l|}{ Severity, n (\%) } \\
\hline Mild & $2(3.0)$ & NA & NA \\
\hline Moderate & $41(62.1)$ & NA & NA \\
\hline Sever & $23(34.8)$ & NA & NA \\
\hline
\end{tabular}

Values are presented as mean (standard deviation) or $n$ (\%). COVID, coronavirus disease; BMI, body mass index; NA, not applicable.

Table 3 Characteristics of quantitative airway dimensions of patients with COVID-19 who had paired CT scans versus non-COVID-19 participants

\begin{tabular}{|c|c|c|c|}
\hline Airway dimensions & \multicolumn{2}{|c|}{ COVID-19 } & Non-COVID-19 $(n=32)$ \\
\hline 1st gen. WA $(\%)^{\dagger \ddagger \S}$ & $46.59(2.80)$ & $45.49(2.43)$ & $44.39(2.03)$ \\
\hline 2nd gen. WA $(\%)^{\dagger £ \S}$ & $51.19(2.78)$ & $50.03(2.69)$ & $48.50(2.61)$ \\
\hline 3rd gen. WA $(\%)^{\dagger \ddagger \S}$ & 59.85 (3.39) & $58.13(3.51)$ & $56.12(3.71)$ \\
\hline 5th gen. WA $(\%)^{\dagger \downarrow \S}$ & $67.84(2.28)$ & $66.38(1.94)$ & $64.74(2.73)$ \\
\hline 6th gen. WA $(\%)^{\dagger \ddagger}$ & $69.02(2.08)$ & $67.53(1.70)$ & $66.79(2.64)$ \\
\hline 7th gen. WA $(\%)^{\dagger \ddagger \S}$ & $69.84(2.02)$ & $68.33(2.38)$ & $67.08(2.28)$ \\
\hline 8th gen. WA $(\%)^{\dagger \ddagger \S}$ & $70.21(3.10)$ & $68.99(1.99)$ & $67.10(2.81)$ \\
\hline
\end{tabular}

Values are presented as mean (standard deviation). ${ }^{\dagger}$, denotes $\mathrm{P}<0.05$ by paired-sample $t$-test for comparison between the initial stage and convalescent stage; ${ }^{\ddagger}$, denotes $\mathrm{P}<0.05$ by two independent-sample $t$-test for comparison between the initial stage and non-COVID-19 controls; ${ }^{\S}$, denotes $\mathrm{P}<0.05$ by two independent-sample $t$-test for comparison between the convalescent stage and non-COVID-19 controls. WA\%, wall area fraction; gen., generation; Pi10, internal airway perimeter of $10 \mathrm{~mm}$.

also shown between these two groups, and no significant difference existed regarding the WA\% of the $1^{\text {st }}$ to $8^{\text {th }}$ generations of airways and Pi10. Considering disease severity may also have an impact on airway wall dimensions, patients who were diagnosed as having severe pneumonia were compared with mild/moderate cases. Compared to the mild/moderate-subgroup, patients in the severe subgroup were on average older ( 45.87 versus 56.58 years, $\mathrm{P}=0.001$ ) and had a longer duration of hospitalization (21.34 versus
27.54 days, $\mathrm{P}=0.003)$. Generally, both the WA\% and Pi10 showed no meaningful difference between severe cases and mild/moderate cases.

\section{Effect of clinical factors on airway dimensions in patients with COVID-19}

To explore the association between airway wall dimensions and certain potential clinical factors, such as duration of 

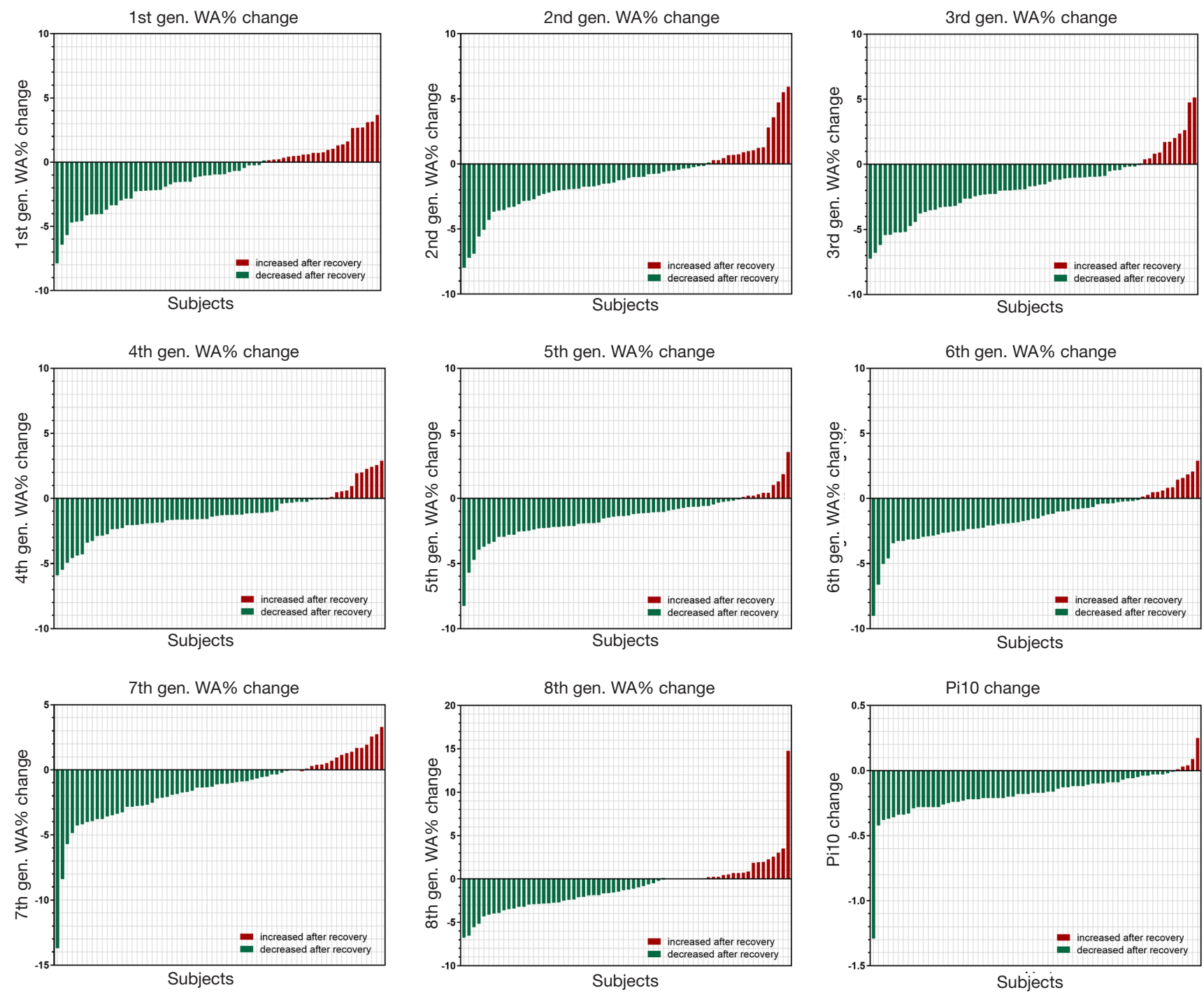

Figure 1 Waterfall plots illustrate changes in the WA\% of the $1^{\text {st }}$ to $8^{\text {th }}$ generation of airway and the Pi10 in 66 individual patients with COVID-19 who had paired CT scans during the disease course. The majority of the patients with COVID-19 had decreased WA\% and Pi10 at the convalescent stage, compared to that at the initial stage. WA\%, wall area fraction; gen., generation; Pi10, internal airway perimeter of $10 \mathrm{~mm}$. WA\%, percentage of wall area; COVID-19, coronavirus disease 19.

hospitalization and disease severity, linear regression models were constructed. In univariate linear regression analysis, increasing duration of hospitalization was negatively associated with the WA\% of the $1^{\text {st }}$ to $6^{\text {th }}$ generation of airway, but no statistically significant association was found between duration of hospitalization and Pi10 (Table 5). After adjusting for age, sex and smoking status, such a relationship between WA\% and Pi10 remained concordant (Table 6). Generally, WA\% decreased by $0.06 \%$ to $0.16 \%$ with every day spent in the duration of hospitalization. This outcome echoes the pattern noted in the comparison between quantitative CT characteristics in disease initiation and early convalescence, reflecting that the thickening of airway wall dimensions in patients with COVID-19 tends to decrease to normal when the human body recovers. Univariate regression analysis demonstrated that the relationship between severity of disease and the WA\% of the $1^{\text {st }}$ to $8^{\text {th }}$ generation airways is not clear. After being 
Table 4 Comparison of inner area $\left(\mathrm{mm}^{2}\right)$ of $1^{\text {st }}$ to $8^{\text {th }}$ generations of airways between patients with COVID-19 and non-COVID-19 controls

\begin{tabular}{|c|c|c|c|}
\hline Inner area $\left(\mathrm{mm}^{2}\right)$ & \multicolumn{2}{|c|}{ COVID-19 patients } & Non-COVID-19 controls $(n=32)$ \\
\hline 1st gen. ${ }^{\dagger}$ & 121.05 (39.95) & $131.21(37.71)$ & $122.54(40.32)$ \\
\hline 2nd gen. ${ }^{\dagger}$ & $69.32(20.46)$ & 77.64 (22.37) & $73.59(25.50)$ \\
\hline $3 r d$ gen. $^{\dagger}$ & 34.91 (11.78) & 38.07 (11.98) & $39.75(20.11)$ \\
\hline 5th gen. ${ }^{\ddagger}$ & $10.18(3.03)$ & $10.97(3.72)$ & $12.46(5.61)$ \\
\hline 6th gen. & $7.89(2.24)$ & $7.93(2.14)$ & $8.81(3.71)$ \\
\hline 7th gen. & 7.09 (1.99) & $10.41(28.59)$ & $8.38(5.44)$ \\
\hline 8th gen. ${ }^{\S}$ & $7.48(5.78)$ & $6.70(2.03)$ & $7.90(3.61)$ \\
\hline
\end{tabular}

Values are presented as mean (standard deviation). ${ }^{\dagger}$, denotes $\mathrm{P}<0.05$ by paired-sample $t$-test for comparison between the initial stage and convalescence; ${ }^{\ddagger}$, denotes $\mathrm{P}<0.05$ by two independent-sample $t$-test for comparison at the initial stage with non-COVID- 19 controls; $\S$, denotes $\mathrm{P}<0.05$ by two independent-sample $t$-test for comparison in convalescence with non-COVID-19 controls. gen., generation; COVID-19, 2019-coronavirus infected disease.

adjusted for age, sex and smoking status, multivariate linear regression analysis showed neither WA\% nor Pi10 was associated with disease severity.

To further investigate whether airway wall thickening was due to inflammation, the correlations between airway wall thickening (WA\% and Pi10) and the level of inflammatory indicators during the disease course were analyzed using partial correlation analysis. Results are shown in Tables 7 and 8 . The levels of CRP and serum amyloid A (SAA) during COVID-19 were not associated with airway wall thickening in either the initial stage or in convalescence. Such results are consistent with the dissociation of airway dimensions and disease severity. Together with the non-significant correlation of inner area between patients with COVID-19 and non-COVID-19 participants, these results indicate that, although airway wall thickened in patients with COVID-19, the airways are not the part of the lung that is directly attacked and most affected by COVID-19 (like the pulmonary vessels), but more of a collateral change of edema.

\section{Discussion}

This investigation described the characteristics of airway wall dimensions in patients with COVID-19 using quantitative CT metrics, with the purpose to provide evidence of whether airway thickening occurs in patients with COVID-19 during early recovery. By retrospective analysis of imaging data using quantitative CT measurements, we demonstrated that airway dimensions are significantly greater in patients with COVID-19 during the course of the disease. These structural changes indicate bronchial edema was caused by increased vascular permeability. A longer duration of hospitalization is associated with a decreased percentage of airway wall area, reflecting the improving trend of airway edema during the disease recovery process. No evidence shows that disease severity or inflammatory markers (such as CRP and SAA) are associated with airway dimensions, suggesting that the bronchial thickening is more likely a result of vascular congestion rather than a targeted lesion due to inflammation. These results support our hypothesis that airway wall thickening exists in patients with COVID-19 as a nonspecific change due to pulmonary inflammatory congestion, even during early convalescence.

The changes in airway wall thickness in patients with COVID-19 described in this article display similarity with other reports. Many studies have described the bronchial changes following SARS-CoV-2 infection observed on autopsy, CT imaging and in spirometry. Autopsy studies have shown that in some patients, apart from pronounced diffuse alveolar damage (DAD) that indicates acute respiratory distress syndrome (ARDS) $(6,29)$, excessive mucus secretion causes bronchial thickening and mucus plugs within the airways $(8,30)$. In an analysis of pulmonary function in patients with COVID-19, forced expiratory 
Table 5 Univariate linear regression models of the impact of duration of hospitalization and severity on quantitative CT-based airway dimension in patients with COVID-19 at the convalescent stage

\begin{tabular}{|c|c|c|c|c|}
\hline Variables & $\beta$ & \multicolumn{2}{|c|}{$95 \% \mathrm{Cl}$} & $P$ value \\
\hline \multicolumn{5}{|c|}{ Duration of hospitalization } \\
\hline 1st gen. WA $(\%)^{\dagger}$ & -0.0010 & -0.0017 & -0.0003 & 0.004 \\
\hline 2nd gen. WA $(\%)^{\dagger}$ & -0.0013 & -0.0020 & -0.0006 & 0.001 \\
\hline 4th gen. WA $(\%)^{\dagger}$ & -0.0012 & -0.0018 & -0.0006 & 0.000 \\
\hline 5th gen. WA $(\%)^{\dagger}$ & -0.0010 & -0.0015 & -0.0005 & 0.000 \\
\hline 6 th gen. WA $(\%)^{\dagger}$ & -0.0008 & -0.0013 & -0.0004 & 0.000 \\
\hline 7th gen. WA $(\%)^{\dagger}$ & -0.0005 & -0.0012 & 0.0002 & 0.133 \\
\hline \multicolumn{5}{|l|}{ Severity } \\
\hline 1st gen. WA $(\%)^{\S}$ & -0.0121 & -0.0241 & -0.0001 & 0.049 \\
\hline 2nd gen. WA $(\%)^{\S}$ & -0.0107 & -0.0241 & 0.0028 & 0.118 \\
\hline 3rd gen. WA $(\%)^{\S}$ & -0.0175 & -0.0344 & -0.0006 & 0.043 \\
\hline 4th gen. WA $(\%)^{\S}$ & -0.0151 & -0.0272 & -0.0029 & 0.016 \\
\hline 5th gen. WA $(\%)^{\S}$ & -0.0076 & -0.0171 & 0.0020 & 0.119 \\
\hline 6 th gen. WA $(\%)^{\S}$ & -0.0063 & -0.0148 & 0.0021 & 0.140 \\
\hline 7th gen. WA $(\%)^{\S}$ & 0.0016 & -0.0105 & 0.0136 & 0.796 \\
\hline
\end{tabular}

Sample size of the models: ${ }^{\dagger}$, denotes $n=68 ;{ }^{\ddagger}$, denotes $n=67 ;{ }^{\S}$, denotes $n=69$. Cl, confidence interval; WA\%, wall area fraction; gen., generation; Pi10, internal airway perimeter of $10 \mathrm{~mm}$.

volume in the first second (FEV1), forced vital capacity (FVC) and their ratio (FEV1/FVC) were found to be abnormal in $13.6 \%, 9.1 \%$ and $4.5 \%$, respectively (31). Disease severity has no association with these ventilatory abnormalities, which is consistent with our results.

Although thickened during COVID-19 infection, the airway wall was not the primary targeted part in the lung. As is shown in our study, the thickening of the airway wall was correlated with neither the level of inflammatory indicators nor disease severity. This is because the thickening of the airway wall is probably one of the manifestations of pulmonary inflammatory congestion. Given that bronchial edema is not a primary lesion due to inflammation (such as bronchiectasis), it is reasonable that airway measures (airway wall area and inner area) are not correlated with inflammatory markers such as CRP and SAA or disease severity. This theory is supported by many other reports. An autopsy study of patients who died from COVID-19 demonstrated severe endothelial injury of the pulmonary vessels (32). SARS-CoV-2 infects the human body and binds with angiotensin-converting enzyme 2 (ACE 2) (33), resulting in coagulation pathway activation and increased vascular permeability (34). In the convalescence stage of COVID-19 infection, patients' lung diffusing capacity measured by carbon monoxide (DLCO) was found impaired (35). These results indicate that the pulmonary interstitium, and not the airways, is the most affected component of the lung during COVID-19 infection. SARS- 
Table 6 Multivariate linear regression models of the impact of duration of hospitalization and severity on quantitative CT-based airway dimension in patients with COVID-19 at the convalescent stage

\begin{tabular}{|c|c|c|c|c|c|c|}
\hline Variables & $\beta$ & \multicolumn{2}{|c|}{$95 \% \mathrm{Cl}$} & $P$ value for $\beta$ & Adjusted $\mathrm{R}^{2}$ & $\begin{array}{l}P \text { value for } \\
\text { adjusted } R^{2}\end{array}$ \\
\hline \multicolumn{7}{|c|}{ Duration of hospitalization } \\
\hline 1st gen. WA (\%) ${ }^{\dagger}$ & -0.0007 & -0.0014 & 0.0000 & 0.051 & 0.164 & 0.004 \\
\hline 2nd gen. WA (\%) ${ }^{\dagger}$ & -0.0012 & -0.0019 & -0.0004 & 0.002 & 0.213 & 0.001 \\
\hline 4th gen. WA $(\%)^{\dagger}$ & -0.0012 & -0.0018 & -0.0005 & 0.001 & 0.227 & $<0.001$ \\
\hline 5th gen. WA $(\%)^{\dagger}$ & -0.0009 & -0.0014 & -0.0004 & 0.001 & 0.271 & $<0.001$ \\
\hline 6th gen. WA $(\%)^{\dagger}$ & -0.0008 & -0.0012 & -0.0003 & 0.001 & 0.245 & $<0.001$ \\
\hline 7 th gen. WA $(\%)^{\dagger}$ & -0.0006 & -0.0013 & 0.0001 & 0.098 & 0.066 & 0.080 \\
\hline \multicolumn{7}{|l|}{ Severity } \\
\hline 1st gen. WA $(\%)^{\S}$ & -0.0054 & -0.0182 & 0.0073 & 0.400 & 0.119 & 0.016 \\
\hline 2nd gen. WA $(\%)^{\S}$ & -0.0080 & -0.0223 & 0.0063 & 0.268 & 0.097 & 0.032 \\
\hline 3rd gen. WA $(\%)^{\S}$ & -0.0173 & -0.0354 & 0.0008 & 0.061 & 0.109 & 0.022 \\
\hline 4th gen. WA $(\%)^{\S}$ & -0.0151 & -0.0280 & -0.0021 & 0.023 & 0.134 & 0.010 \\
\hline 5th gen. WA $(\%)^{\S}$ & -0.0061 & -0.0162 & 0.0040 & 0.231 & 0.116 & 0.018 \\
\hline 6 th gen. WA $(\%)^{\S}$ & -0.0052 & -0.0141 & 0.0038 & 0.253 & 0.106 & 0.024 \\
\hline 7th gen. WA $(\%)^{\S}$ & 0.0007 & -0.0125 & 0.0138 & 0.920 & 0.007 & 0.358 \\
\hline
\end{tabular}

All models adjusted for sex, age and smoking status. Sample size of the models: ${ }^{\dagger}$, denotes $n=68 ;{ }^{\ddagger}$, denotes $n=67 ;{ }^{\S}$, denotes $n=69$. Cl, confident interval; WA\%, wall area fraction; gen., generation; Pi10, internal airway perimeter of $10 \mathrm{~mm}$.

CoV-2 primarily damages pulmonary vessels, causes airway wall swelling along the way and results in pulmonary interstitial and alveolar edema in the end.

Various pulmonary abnormalities could occur during and after pneumonia, such as post-inflammatory pulmonary fibrosis (PPF) and organizing pneumonia (36). SARS$\mathrm{CoV}-2$, as a family member of Betacoronavirus, resembles SARS-CoV and MERS-CoV in many ways. For example, both SARS and MERS can cause ARDS and fibrinous exudate, leading to lung fibrosis and pulmonary function impairment, especially diffusion capacity (37-39). One prior study revealed that impaired diffusion capacity and restrictive ventilatory defect could continue to exist in patients with COVID-19 after discharge and that such impaired lung function correlates with a more severe condition (31). Such structural and functional impairments may persist for years after recovery, or the patient may never fully recover $(21,40,41)$. Before our study, there has been no report focusing on airway dimension changes after SARS-CoV-2 infection. Since our understanding of SARSCoV-2 is limited, whether airway wall thickening could be long-term and how it impacts pulmonary function also need our attention.

Several limitations of our study should be addressed. Firstly, no critical cases were included in our study because critically ill patients with COVID-19 were transferred to a designated hospital for further advanced treatment. Secondly, information regarding airway wall thickness prior 
Table 7 Partial correlation coefficient between the highest level of inflammatory indicators during the disease course and wall area of $1^{\text {st }}-8^{\text {th }}$ generations of airways in convalescence

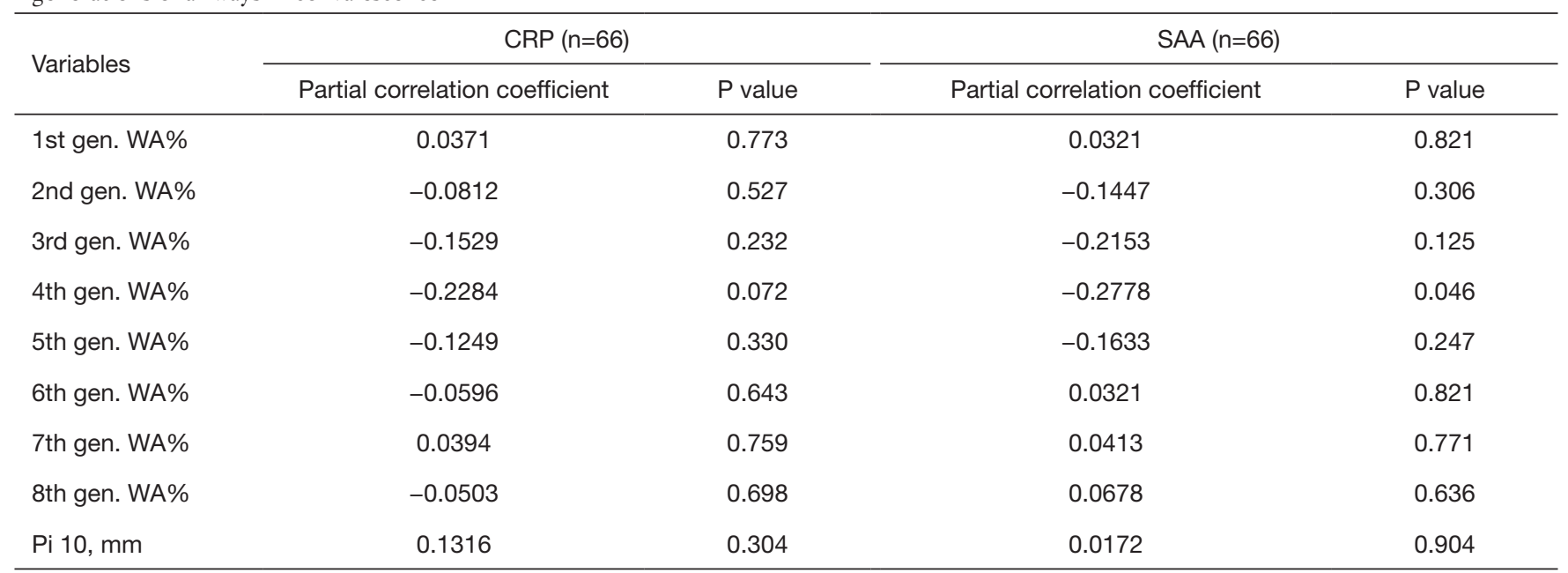

WA\%, wall area fraction; gen., generation; Pi10, internal airway perimeter of $10 \mathrm{~mm}$. Adjusted for age, sex, smoking status. CRP, C-reactive protein; SAA, serum amyloid A.

Table 8 Partial correlation coefficient between the level of inflammatory indicators during the disease course and wall area of $1^{\text {st }}-8^{\text {th }}$ generations of airways at the initial stage

\begin{tabular}{|c|c|c|c|c|}
\hline Variables & \multicolumn{2}{|l|}{ CRP $(n=66)$} & \multicolumn{2}{|c|}{ SAA $(n=66)$} \\
\hline 1st gen. WA\% & -0.0175 & 0.892 & -0.0579 & 0.684 \\
\hline 2nd gen. WA\% & -0.1148 & 0.370 & -0.1009 & 0.477 \\
\hline 3rd gen. WA\% & -0.1832 & 0.151 & -0.0812 & 0.567 \\
\hline 5th gen. WA\% & -0.0355 & 0.782 & -0.0238 & 0.867 \\
\hline 6th gen. WA\% & -0.0237 & 0.853 & 0.0834 & 0.556 \\
\hline 7th gen. WA\% & 0.0970 & 0.457 & 0.1905 & 0.185 \\
\hline 8th gen. WA\% & -0.1731 & 0.206 & 0.0607 & 0.692 \\
\hline
\end{tabular}

Adjusted for age, sex, smoking status. CRP, C-reactive protein; SAA, serum amyloid A. WA\%, wall area fraction; gen., generation; Pi10, internal airway perimeter of $10 \mathrm{~mm}$.

to patient infection with SARS-CoV-2 was unavailable, so we could not perform that self-comparison. However, given that most of our patients were non-smokers or were without baseline pulmonary diseases, comparison analysis between patients and non-COVID-19 participants could indirectly represent self-comparison before and after infection.

In conclusion, our findings demonstrate that airway wall dimensions in patients with COVID-19 are greater than in non-COVID-19 individuals. However, the probable effect of interstitial fibrosis on airway dilation is greater than the effect of airway wall thickening on airway stenosis, resulting in a greater effect on impaired diffusion capacity than decreased ventilation function. Therefore, physician should prioritize improving the diffusion capacity over the ventilation capacity when using mechanical ventilation to alleviate dyspnea for patients with COVID-19. Given the 
fact that SARS-CoV-2 can cause histopathologic and clinical changes similar to SARS-CoV and MERS-CoV, PPF and its effect on pulmonary structural as well as functional damage as a disease sequela is most concerning. Whether this kind of structural airway change persists in the long run after recovery from COVID-19, causing irreversible change in pulmonary function, is not yet clear. Longitudinal analysis with a larger sample size is needed to answer this question.

\section{Acknowledgments}

Funding: This work was supported by the National Key Research and Development Program of China (2018YFC1311900).

\section{Footnote}

Reporting Checklist: The authors have completed the STROBE reporting checklist. Available at http://dx.doi. org/10.21037/jtd-20-2790

Data Sharing Statement: Available at http://dx.doi. org/10.21037/jtd-20-2790

Conflicts of Interest: All authors have completed the ICMJE uniform disclosure form (available at http://dx.doi. org/10.21037/jtd-20-2790). The authors have no conflicts of interest to declare.

Ethical Statement: The authors are accountable for all aspects of the work in ensuring that questions related to the accuracy or integrity of any part of the work are appropriately investigated and resolved. The study was conducted in accordance with the Declaration of Helsinki (as revised in 2013). The study was approved by the Institutional Review Board at both Guangzhou Eighth People's Hospital and the First Affiliated Hospital of Guangzhou Medica University (Ethics No. 20201134). Because of the retrospective nature of the research, written informed consent for data collection was waived for all participants.

Open Access Statement: This is an Open Access article distributed in accordance with the Creative Commons Attribution-NonCommercial-NoDerivs 4.0 International License (CC BY-NC-ND 4.0), which permits the noncommercial replication and distribution of the article with the strict proviso that no changes or edits are made and the original work is properly cited (including links to both the formal publication through the relevant DOI and the license). See: https://creativecommons.org/licenses/by-nc-nd/4.0/.

\section{References}

1. Huang C, Wang Y, Li X, et al. Clinical features of patients infected with 2019 novel coronavirus in Wuhan, China. Lancet 2020;395:497-506.

2. Lu R, Zhao X, Li J, et al. Genomic characterisation and epidemiology of 2019 novel coronavirus: implications for virus origins and receptor binding. Lancet 2020;395:565-74.

3. Li Q, Guan X, Wu P, et al. Early transmission dynamics in Wuhan, China, of novel coronavirus-infected pneumonia. N Engl J Med 2020;382:1199-207.

4. Zhu N, Zhang D, Wang W, et al. A novel coronavirus from patients with pneumonia in China, 2019. N Engl J Med 2020;382:727-33.

5. World Health Organization. Coronavirus disease (COVID-19) situation report Available online: https://www.who.int/emergencies/diseases/novelcoronavirus-2019/situation-reports

6. Xu Z, Shi L, Wang Y, et al. Pathological findings of COVID-19 associated with acute respiratory distress syndrome. Lancet Respir Med 2020;8:420-2.

7. Zhou F, Yu T, Du R, et al. Clinical course and risk factors for mortality of adult inpatients with COVID-19 in Wuhan, China: a retrospective cohort study. Lancet 2020;395:1054-62.

8. Diagnosis and treatment protocol for novel coronavirus pneumonia (trial version 7). Chin Med J (Engl) 2020;133:1087-95.

9. Kanne JP, Little BP, Chung JH, et al. Essentials for Radiologists on COVID-19: An Update- Radiology Scientific Expert Panel. Radiology 2020;296:E113-E114.

10. Fang Y, Zhang H, Xie J, et al. Sensitivity of chest CT for COVID-19: Comparison to RT-PCR. Radiology 2020;296:E115-E117.

11. American College of Radiology (ACR). ACR Recommendations for the use of chest radiography and computed tomography (CT) for suspected COVID-19 Infection. Available online: https://www.acr.org/ Advocacy-and-Economics/ACR-Position-Statements/ Recommendationsfor-Chest-Radiography-and-CT-forSuspected-COVID19-Infection. Published March 11, 2020. Accessed April 1, 2020.

12. Lv M, Wang M, Yang N, et al. Chest computed 
tomography for the diagnosis of patients with coronavirus disease 2019 (COVID-19): a rapid review and metaanalysis. Ann Transl Med 2020;8:622.

13. Hui TCH, Khoo HW, Young BE, et al. Clinical utility of chest radiography for severe COVID-19. Quant Imaging Med Surg 2020;10:1540-50.

14. $\mathrm{Xu} \mathrm{X}, \mathrm{Yu} \mathrm{C}, \mathrm{Qu} \mathrm{J}$, et al. Imaging and clinical features of patients with 2019 novel coronavirus SARS-CoV-2. Eur J Nucl Med Mol Imaging 2020;47:1275-80.

15. Guan WJ, Ni ZY, Hu Y, et al. Clinical characteristics of coronavirus disease 2019 in China. N Engl J Med 2020;382:1708-20.

16. Wang Y, Dong C, Hu Y, et al. Temporal changes of CT findings in 90 patients with COVID-19 pneumonia: A longitudinal study. Radiology 2020;296:E55-E64.

17. Yang Y, Peng F, Wang R, et al. The deadly coronaviruses: The 2003 SARS pandemic and the 2020 novel coronavirus epidemic in China. J Autoimmun 2020;109:102434.

18. Lee $\mathrm{N}$, Hui D, Wu A, et al. A major outbreak of severe acute respiratory syndrome in Hong Kong. N Engl J Med 2003;348:1986-94.

19. Assiri A, Al-Tawfiq JA, Al-Rabeeah AA, et al. Epidemiological, demographic, and clinical characteristics of 47 cases of middle east respiratory syndrome coronavirus disease from Saudi Arabia: a descriptive study. Lancet Infect Dis 2013;13:752-61.

20. Zaki AM, van Boheemen S, Bestebroer TM, et al. Isolation of a novel coronavirus from a man with pneumonia in Saudi Arabia. N Engl J Med 2012;367:1814-20.

21. Park WB, Jun KI, Kim G, et al. Correlation between pneumonia severity and pulmonary complications in middle east respiratory syndrome. J Korean Med Sci 2018;33:e169.

22. Hsu HH, Tzao C, Wu CP, et al. Correlation of highresolution CT, symptoms, and pulmonary function in patients during recovery from severe acute respiratory syndrome. Chest 2004;126:149-58.

23. Chang YC, Yu CJ, Chang SC, et al. Pulmonary sequelae in convalescent patients after severe acute respiratory syndrome: evaluation with thin-section CT. Radiology 2005;236:1067-75.

24. Antonio GE, Wong KT, Hui DS, et al. Thin-section CT in patients with severe acute respiratory syndrome following hospital discharge: preliminary experience. Radiology 2003;228:810-5.

25. Estépar RS, Ross JC, Kindlmann GL, et al. Automatic airway analysis for genome-wide association studies in COPD. Proc IEEE Int Symp Biomed Imaging
2012:1467-70.

26. Grydeland TB, Dirksen A, Coxson HO, et al. Quantitative computed tomography measures of emphysema and airway wall thickness are related to respiratory symptoms. Am J Respir Crit Care Med 2010;181:353-9.

27. Nakano Y, Muro S, Sakai H, et al. Computed tomographic measurements of airway dimensions and emphysema in smokers. Correlation with lung function. Am J Respir Crit Care Med 2000;162:1102-8.

28. Martinez CH, Chen YH, Westgate PM, et al. Relationship between quantitative CT metrics and health status and BODE in chronic obstructive pulmonary disease. Thorax 2012;67:399-406.

29. Wichmann D, Sperhake JP, Lütgehetmann M, et al. Autopsy Findings and Venous Thromboembolism in Patients With COVID-19: A Prospective Cohort Study. Ann Intern Med 2020;173:268-77.

30. Fox SE, Akmatbekov A, Harbert JL, et al. Pulmonary and cardiac pathology in African American patients with COVID-19: an autopsy series from New Orleans. Lancet Respir Med 2020;8:681-6.

31. Mo X, Jian W, Su Z, et al. Abnormal pulmonary function in COVID-19 patients at time of hospital discharge. Eur Respir J 2020;5 5:2001217.

32. Ackermann M, Verleden SE, Kuehnel M, et al. Pulmonary Vascular Endothelialitis, Thrombosis, and Angiogenesis in Covid-19. N Engl J Med 2020;383:120-8.

33. Hoffmann M, Kleine-Weber H, Schroeder S, et al. SARSCoV-2 Cell Entry Depends on ACE2 and TMPRSS2 and Is Blocked by a Clinically Proven Protease Inhibitor. Cell 2020;181:271-280.e8.

34. Teuwen LA, Geldhof V, Pasut A, Carmeliet P. COVID-19: the vasculature unleashed. Nat Rev Immunol 2020;20:389-91.

35. Huang $\mathrm{Y}$, Tan $\mathrm{C}, \mathrm{Wu} \mathrm{J}$, et al. Impact of coronavirus disease 2019 on pulmonary function in early convalescence phase. Respir Res 2020;21:163.

36. Kligerman SJ, Franks TJ, Galvin JR. From the radiologic pathology archives: organization and fibrosis as a response to lung injury in diffuse alveolar damage, organizing pneumonia, and acute fibrinous and organizing pneumonia. Radiographics 2013;33:1951-75.

37. Venkataraman T, Frieman MB. The role of epidermal growth factor receptor (EGFR) signaling in SARS coronavirus-induced pulmonary fibrosis. Antiviral Res 2017;143:142-50.

38. Ding Y, Wang H, Shen H, et al. The clinical pathology of severe acute respiratory syndrome (SARS): a report from 
China. J Pathol 2003;200:282-9.

39. $\mathrm{Ng} \mathrm{DL}, \mathrm{Al}$ Hosani F, Keating MK, et al. Clinicopathologic, Immunohistochemical, and Ultrastructural Findings of a Fatal Case of Middle East Respiratory Syndrome Coronavirus Infection in the United Arab Emirates, April 2014. Am J Pathol 2016;186:652-8.

40. Ngai JC, Ko FW, Ng SS, et al. The long-term impact

Cite this article as: $\mathrm{Xu} \mathrm{J}$, Liang Z, Jian W, Li J, Tang G, Mo X, Zhang D, Zheng J, Qian Y, Liu J, Li S. Changes of quantitative CT-based airway wall dimensions in patients with COVID-19 during early recovery. J Thorac Dis 2021;13(3):1517-1530. doi: $10.21037 /$ jtd-20-2790 of severe acute respiratory syndrome on pulmonary function, exercise capacity and health status. Respirology 2010;15:543-50.

41. Hui DS, Wong KT, Ko FW, et al. The 1-year impact of severe acute respiratory syndrome on pulmonary function, exercise capacity, and quality of life in a cohort of survivors. Chest 2005;128:2247-61. 